\title{
Local thermal equilibrium of strange hadrons at CBM energies
}

\author{
Somnath $\mathbf{D e}^{*}$ \\ Institute of Physics, Bhubaneswar, Odisha, India \\ E-mail: somnath.de@iopb.res.in \\ Sudipan De \\ Universidade de São Paulo, São Paulo, Brasil \\ E-mail: sudipaneif.usp.br

\section{Subhasis Chattopadhyay} \\ Variable Energy Cyclotron Centre, 1/AF, Bidhan Nagar, Kolkata, India \\ E-mail: sub@vecc.gov. in
}

The Compressed Baryonic Matter (CBM) experiment at FAIR/ GSI laboratory is being designed to perform heavy-ion collisions in fixed target mode at beam energies 5-45 GeV per nucleon. One of the major goal of the experiment is to explore the properties of matter at high baryon density and moderate temperature. Thus an important question arises whether the dense hadronic matter created in such collisions will achieve a local thermal equilibrium, in particular the strange hadrons. We have investigated the conditions of local thermal equilibrium of strange, non-strange baryons and strange mesons in a small element of volume in the rapidity window $|y|<1.0$ for $\mathrm{Au}+\mathrm{Au}$ collisions at laboratory energy 10, 20,30, $40 \mathrm{GeV}$ per nucleon. The microscopic transport model UrQMD-3.3p2 has been used for this purpose. We have studied the time evolution of density, longitudinal-to-transverse pressure anisotropy and inverse slope parameter of the energy spectra of non-strange baryons, strange baryons and mesons inside the cell. The strangeness content is dominated by baryons at all energies, however contribution from mesons become significant at higher energies. The pressure isotropization time of non-strange and strange baryons are found nearly similar and decreases with increase in beam energy. We found that the baryons (strange and non-strange) and strange mesons attain thermal equilibrium for time $>9 \mathrm{fm} / \mathrm{c}$ at $10 \mathrm{~A}$ $\mathrm{GeV}$ and for time $>6 \mathrm{fm} / \mathrm{c}$ at $40 \mathrm{~A} \mathrm{GeV}$ laboratory energy.

7th International Conference on Physics and Astrophysics of Quark Gluon Plasma

1-5 February, 2015

Kolkata, India

${ }^{*}$ Speaker. 


\section{Introduction}

Heavy ion collisions at the Large Hadron Collider (LHC) and Relativistic Heavy Ion Collider (RHIC) are involved to study the phase diagram of strongly interacting matter at high temperature and low baryon density. In contrast, the Compressed Baryonic Matter (CBM) experiment $[1,2]$ at the upcoming Facility for Antiproton and Ion Research (FAIR) [3] will explore the phase diagram at moderate temperature and high baryon density. The CBM experiment is designed to study the bulk and rare probes with high luminosity beam in nucleus-nucleus collisions at beam energies 5-45 GeV per nucleon [2, 4].

Macroscopic and microscopic models are needed to understand the dynamic properties of the matter created during such collisions. A question of great importance in these studies is whether local thermodynamic equilibrium of the system is reached or not. This is a crucial point for the existing models. Macroscopic models like hydrodynamics or thermal model adopt local thermal equilibrium as ad hoc assumption. On the other hand the various Monte Carlo microscopic models do not assume the local thermal equilibrium $[5,6]$. Thus it is very interesting to study the criteria of local thermal equilibrium for the heavy ion collisions at CBM experiment by using one particular microscopic model. For this purpose we employed the microscopic N-body transport model called Ultra-relativistic Quantum Molecular Dynamics (UrQMD) [7]. We have investigated the timescale of local thermal equilibration of non-strange and strange baryons in an elementary volume in phase-space from the time evolution of longitudinal-to-transverse pressure anisotropy and slope of the energy spectrum in central $\mathrm{Au}+\mathrm{Au}$ collisions at four incident beam energies (See Ref. [8] for details).

\section{Analysis details}

UrQMD-version: 3.3p2 was used in cascade mode without invoking any hydrodynamic evolution for the initial state. Simulations were done for $\mathrm{Au}-\mathrm{Au}$ collisions of impact parameter $\mathrm{b}=2 \mathrm{fm}$ at laboratory energies, $E_{\text {lab }}=10 \mathrm{~A}, 20 \mathrm{~A}, 30 \mathrm{~A}$ and $40 \mathrm{~A} \mathrm{GeV}$ for different time steps ranging from 1 $\mathrm{fm} / \mathrm{c}$ to $15 \mathrm{fm} / \mathrm{c}$ within $\left|y_{c m}\right|<1.0$. 60K events are analyzed in each time step. The center of mass frame is considered as the computational frame in the analysis. A cell of dimension $2 \times 2 \times 2 \mathrm{fm}^{3}$ about the origin has been chosen to minimize the effect of collective flow on the observables of the system. We calculated the net particle density, different components of microscopic pressure for non-strange baryons, strange baryons and mesons and energy spectra of Protons and Lamdas inside the cell for each time step. The non-strange baryons include Proton $(p)$ and Neutron $(n)$, the strange baryons include Lamda $(\Lambda)$, Sigma $(\Sigma)$, Cascade $(\Xi)$, Omega $(\Omega)$ and the strange mesons include Kaons $\left(K^{+}, K^{0}\right)$. All the higher mass resonances (baryon and meson) are allowed to decay.

\section{Results and discussions}

Figure 1 shows the time evolution of net non-strange baryon density, net strange baryon density, net kaon density, and net strange baryon to kaon ratio at $E_{l a b}=10 \mathrm{~A}, 20 \mathrm{~A}, 30 \mathrm{~A}$ and $40 \mathrm{~A} \mathrm{GeV}$. Here time $(\mathrm{t})$ is the elapsed time in center of mass frame. The maximum of the net particle density has occured around $t=2 R /\left(\gamma_{c m} v_{c m}\right)$, when the two nuclei pass through each other, then falls as the 
system expands. Here $\mathrm{R}$ is the radius of Au nucleus, $\gamma_{c m}$ and $v_{c m}$ are the Lorentz boost and velocity in center of mass frame. Maximum matter density is found around $6 \mathrm{fm} / \mathrm{c}$ at $10 \mathrm{~A} \mathrm{GeV}$ and $3 \mathrm{fm} / \mathrm{c}$ at $40 \mathrm{~A} \mathrm{GeV}$ for all the species. The peak baryonic (non-strange and strange) matter density has been found 7-8 times the ground state nuclear matter density. The time evolution of strange baryon to meson ratio shows the net strangeness content of the created matter is dominated by baryons at all beam energies.
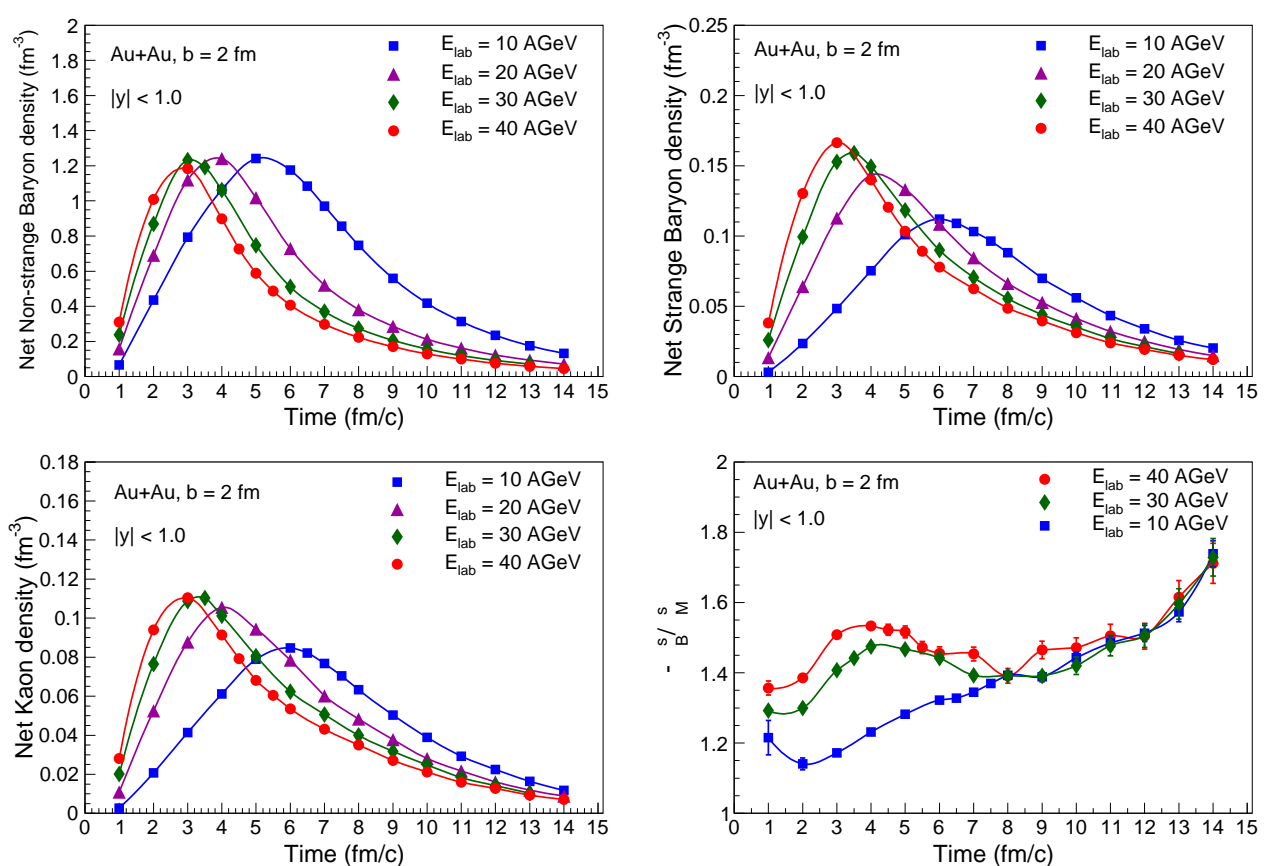

Figure 1: (Color online)(Upper panel) Time evolution of net non-strange baryon density, net strange baryon density, (Lower panel) net kaon density and net strange baryon to kaon ratio inside the central cell for $\mathrm{Au}+\mathrm{Au}$ collisions $(\mathrm{b}=2 \mathrm{fm})$ at the laboratory energies $10 \mathrm{~A}, 20 \mathrm{~A}, 30 \mathrm{~A}, 40 \mathrm{~A} \mathrm{GeV}$. The error bars are statistical only.

Figure 2 shows the longitudinal-to-transverse pressure $\left(P_{L} / P_{T}\right)$ anisotropy at different time steps for non-strange baryons, strange baryons and kaons at $E_{l a b}=10 \mathrm{~A}, 20 \mathrm{~A}, 30 \mathrm{~A}$ and $40 \mathrm{~A} \mathrm{GeV}$. Each plot corresponds to each laboratory energy. The longitudinal and transverse components of microscopic pressure for an ensemble of hadrons are defined as [8]:

$$
P_{L}=\left\langle P_{z}\right\rangle ; \quad P_{T}=\frac{1}{2}\left(\left\langle P_{x}\right\rangle+\left\langle P_{y}\right\rangle\right),
$$

where the $\langle.$.$\rangle corresponds to the statistical average over the number of events.$

Starting from a large value at very beginning of the collisions, $P_{L} / P_{T}$ ratio of baryons reaches unity around $6.5 \mathrm{fm} / \mathrm{c}$ for non-strange baryons and $7 \mathrm{fm} / \mathrm{c}$ for strange baryons at $E_{\text {lab }}=10 \mathrm{~A} \mathrm{GeV}$. However the system evolves further and reaches a constant value $\sim 0.8$ at $\mathrm{t} \geq 9 \mathrm{fm} / \mathrm{c}$. The time denotes the local thermal equilibration time of the system. With increase in beam energy the ratio has become unity earlier and reached a constant value for $\mathrm{t} \geq 8 \mathrm{fm} / \mathrm{c}, \mathrm{t} \geq 7 \mathrm{fm} / \mathrm{c}$ and $\mathrm{t} \geq 6 \mathrm{fm} / \mathrm{c}$ at $E_{\text {lab }}=20,30$ and $40 \mathrm{~A} \mathrm{GeV}$ respectively. The pressure anisotropy $\left(P_{L} / P_{T}\right)$ of kaons are found to 

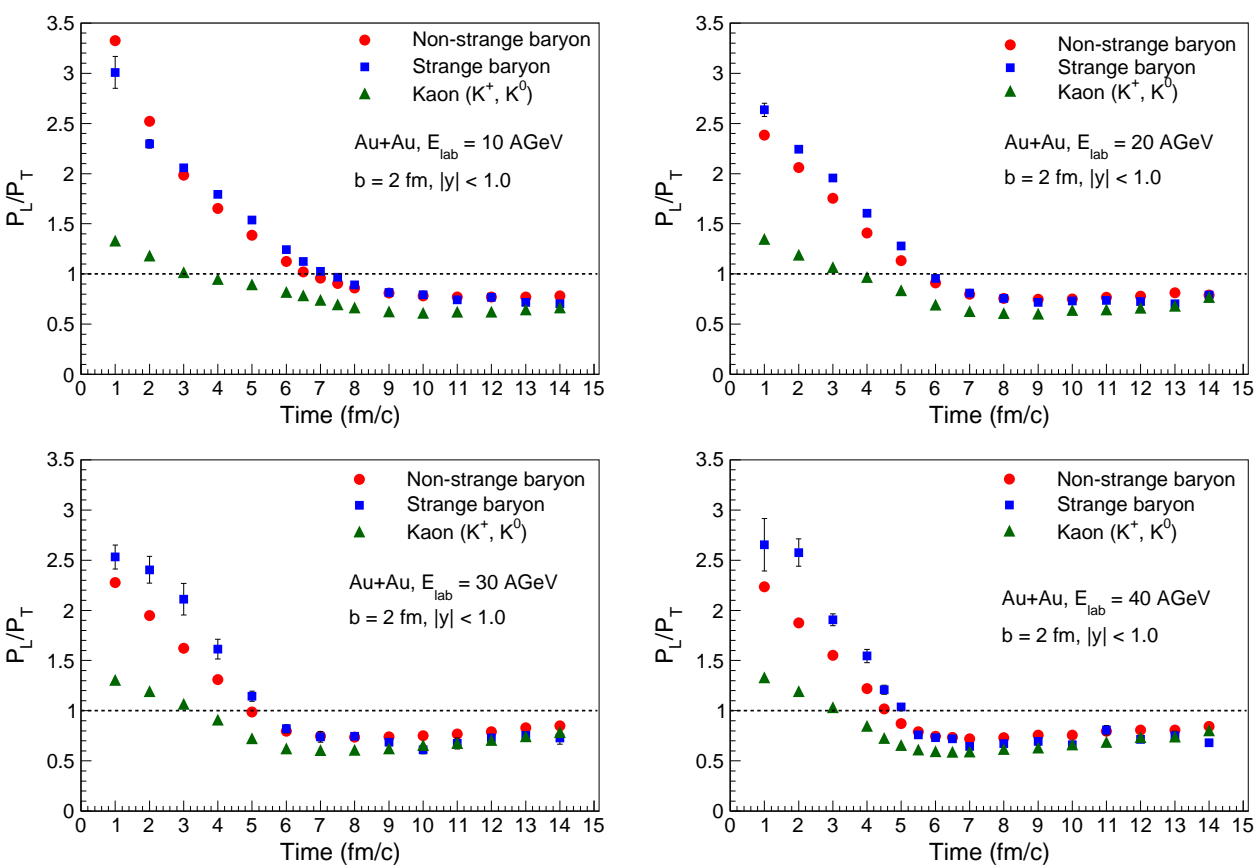

Figure 2: (Color online) Longitudinal-to-transverse pressure ratio $\left(P_{L} / P_{T}\right)$ as a function of time inside the central cell for $\mathrm{Au}+\mathrm{Au}$ collisions $(\mathrm{b}=2 \mathrm{fm})$ at the laboratory energies 10A, 20A, 30A, 40A GeV. Different symbols represent non-strange baryons, strange baryons, kaons. The error bars are statistical only.

smaller than that of baryons as it contains one valance quark of the leading excited hadron. Kaons suffer less interactions in medium and reaches a constant value $\sim 0.6$ at the same time as baryons.

Time evolution of inverse slope parameter $\left(T_{\text {slope }}\right)$ of the energy spectra $\left(E d N / d^{3} p\right.$ vs E) of protons and lamdas inside the cell are depicted in the figure 3. We have fitted the energy spectra by Tsallis distribution [9] and inverse slope parameter of the spectra is given by [10]:

$$
T_{\text {slope }}=T+(q-1) E,
$$

where $E$ is the energy in $\mathrm{GeV}$ and $\mathrm{q}$ is the non-extensive parameter of the Tsallis distribution. The values of $\mathrm{T}$, (q-1) are obtained through fitting the energy spectra upto $\mathrm{E}=3 \mathrm{GeV}$. In the asymptotic limit $E \rightarrow 0$, the $T_{\text {slope }}$ gives the thermodynamic temperature of the system [10]. Results are presented for $\mathrm{E}=0.1 \mathrm{GeV}$, close to the pion mass, at four beam energies. We found that the $T_{\text {slope }}$ approximately scales as $t^{-1 / 3}$ for $\mathrm{t} \gtrsim 9 \mathrm{fm} / \mathrm{c}$ at $10 \mathrm{~A} \mathrm{GeV}$. This indicates an isentropic longitudinal expansion sets in inside the cell at this time. Similar behavior have been found around $t \gtrsim 8 \mathrm{fm} / \mathrm{c}, 6$ $\mathrm{fm} / \mathrm{c}$ and $5 \mathrm{fm} / \mathrm{c}$ at $20 \mathrm{~A} \mathrm{GeV}, 30 \mathrm{~A} \mathrm{GeV}$ and $40 \mathrm{~A} \mathrm{GeV}$ beam energies.

\section{Summary}

We presented the conditions of the local thermal equilibrium of dense hadronic matter created in central $\mathrm{Au}+\mathrm{Au}$ collisions at FAIR proposed laboratory energies 10A, 20A, 30A and 40A GeV. The maximum net baryon density is found at $E_{l a b}=40 \mathrm{~A} \mathrm{GeV}$. It is observed that the net strangeness 

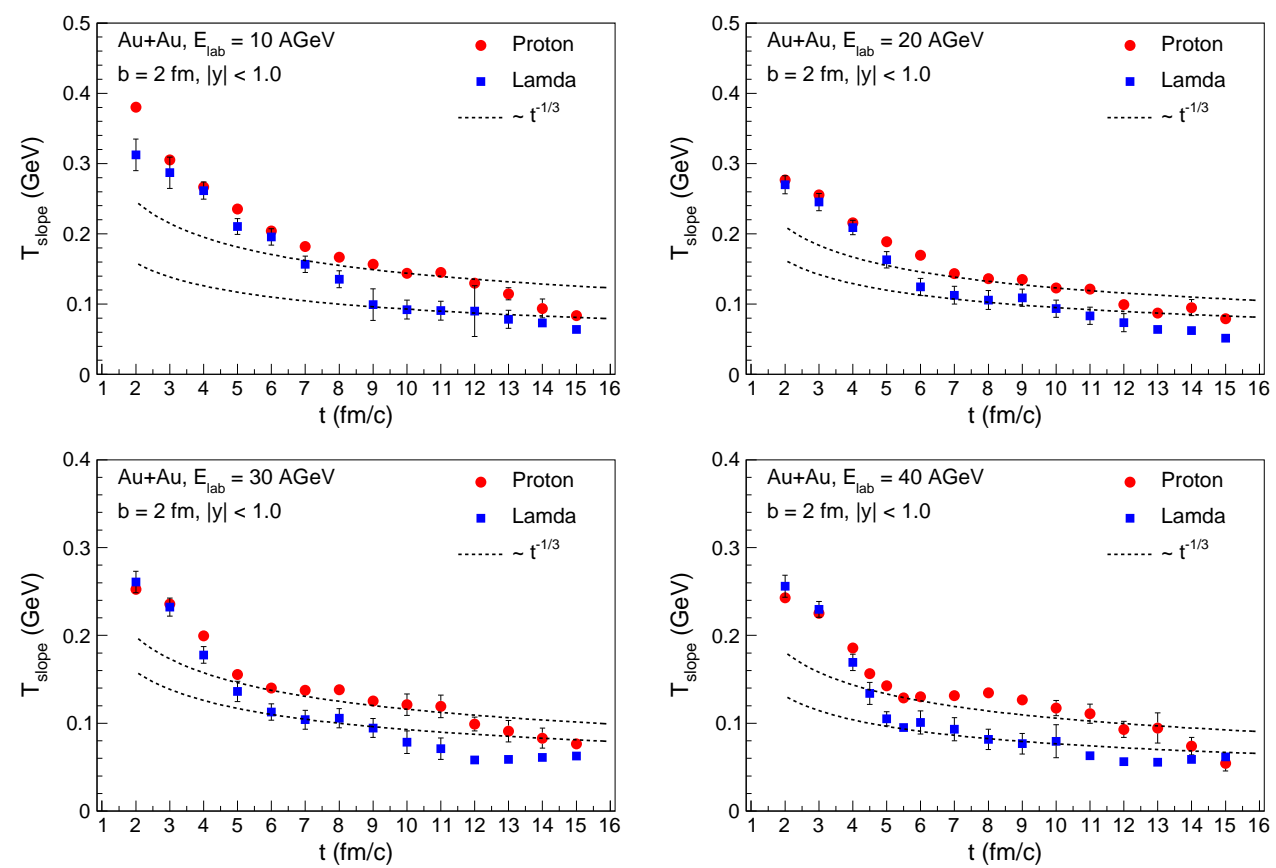

Figure 3: (Color online) Inverse slope parameter $\left(T_{\text {slope }}\right)$ of the energy spectra of protons and lamdas as a function of time steps inside the central cell for central $(b=2 \mathrm{fm}) \mathrm{Au}+\mathrm{Au}$ collisions at the laboratory energies 10A, 20A, 30A, 40A GeV. The error bars are statistical only.

is dominated by the baryons at all energies. Longitudinal-to-transverse pressure anisotropy ratio has become nearly unity at a certain time, which decreases with increasing beam energy. Study of the time evolution of inverse slope parameter $T_{\text {slope }}$ indicates that the system achieve local thermal equilibrium at similar times. Thus the thermalization time of spectra and isotropization time of pressure components are found nearly equal at all corresponding energies.

\section{References}

[1] S. Chattopadhyay, J. Phys. G 35, 104027 (2008).

[2] B. Friman et al. : The CBM Physics Book, Lect. Notes Phys. 814, Springer-Verlag Berlin Heidelberg (2010).

[3] FAIR Baseline Technical Report 2006. http://www.gsi.de/fair/reports/btr.html.

[4] H. R. Schmidt (CBM Collaboration), PoS (Bormio 2013) 061.

[5] W. Cassing, E. L. Bratkovskaya and S. Juchem, Nucl. Phys. A 674, 249 (2000).

[6] Z. W. Lin, C. M. Ko, B. A. Li, B. Zhang and S. Pal, Phys. Rev. C 72, 064901 (2005).

[7] S. A. Bass et al., Prog. Part. Nucl. Phys. 41, 225 (1998); M. Bleicher et al., J. Phys. G 25, 1859 (1999).

[8] S. De, S. De, S. Chattopadhyay, arXiv:1510.01456.

[9] C. Y. Wong and G. Wilk, Acta. Phys. Polon. B 432047 (2012).

[10] T. S. Bíró and G. Purcsel, Phys. Lett. A 372, 1174 (2008). 